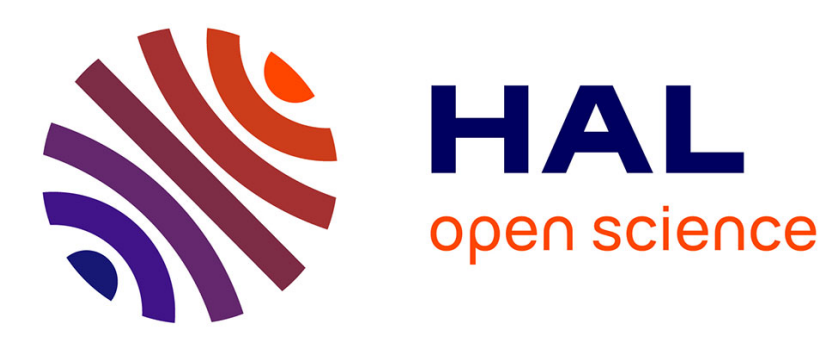

\title{
Continue or Stop Reading? Modeling Decisions in Information Search
}

Francisco Lopez Orozco, Anne Guérin-Dugué, Benoît Lemaire

\section{To cite this version:}

Francisco Lopez Orozco, Anne Guérin-Dugué, Benoît Lemaire. Continue or Stop Reading? Modeling Decisions in Information Search. NLPCS 2012 - 9th International Workshop on Natural Language Processing and Cognitive Science, Jun 2012, Wroclaw, Poland. pp.96-105. hal-00790210

\section{HAL Id: hal-00790210 https://hal.science/hal-00790210}

Submitted on 19 Feb 2013

HAL is a multi-disciplinary open access archive for the deposit and dissemination of scientific research documents, whether they are published or not. The documents may come from teaching and research institutions in France or abroad, or from public or private research centers.
L'archive ouverte pluridisciplinaire HAL, est destinée au dépôt et à la diffusion de documents scientifiques de niveau recherche, publiés ou non, émanant des établissements d'enseignement et de recherche français ou étrangers, des laboratoires publics ou privés. 


\title{
Continue or Stop Reading? Modeling Decisions in Information Search
}

\author{
Francisco López-Orozco ${ }^{1}$, Anne Guérin-Dugué ${ }^{2}$, and Benoît Lemaire ${ }^{1}$ \\ 1 LPNC - University of Grenoble - 38040 Grenoble Cedex 9 - France, \\ \{Francisco.Lopez-Orozco, Benoit.Lemaire\}@upmf-grenoble.fr \\ 2 Gipsa-lab - University of Grenoble - 38042 Grenoble Cedex - France, \\ Anne.Guerin@gipsa-lab.inpg-grenoble.fr
}

\begin{abstract}
This paper presents a cognitive computational model of the way people read a paragraph with the task of quickly deciding whether it is better related to a given goal than another paragraph processed previously. In particular, the model attempts to predict the time at which participants would decide to stop reading the current paragraph because they have enough information to make their decision. We proposed a two-variable linear threshold to account for that decision, based on the rank of the fixation and the difference of semantic similarities between each paragraph and the goal. Our model performance is compared to the eye tracking data of 22 participants.
\end{abstract}

\section{Introduction}

Knowing what web users are doing while they search for information is crucial. Several cognitive models have been proposed to account for some of the processes involved in this activity. Pirolli \& Fu (2003) proposed a model of navigation. Brumby \& Howes (2004) describes how people process information partially in order to select links related to an information goal. Chanceaux et al. (2009) show how visual, semantic and memory processes interact in search tasks.

Information search can be made on any kind of documents, but we are here interested in textual documents, composed of several paragraphs.

Information search is different from pure reading because people have a goal in mind while processing the document. They have to constantly keep in memory this additional information. If the task is only to decide if the current paragraph is related or not to the goal, that paragraph and the goal are the only pieces of information involved. However, in everyday life, people are often concerned with deciding whether the current paragraph is more interesting or not than another one that has been processed previously. For instance, you are looking in a cookbook for a nice French recipe, you already found one but you want to find a better one. In that case, at least three pieces of information have to be together managed in order to make a correct decision: the current paragraph, the goal and a previous paragraph.

This paper attempts to model that particular decision making. It focuses on a behavior that is specific to information search, which is stopping processing a paragraph before it is completely read. 


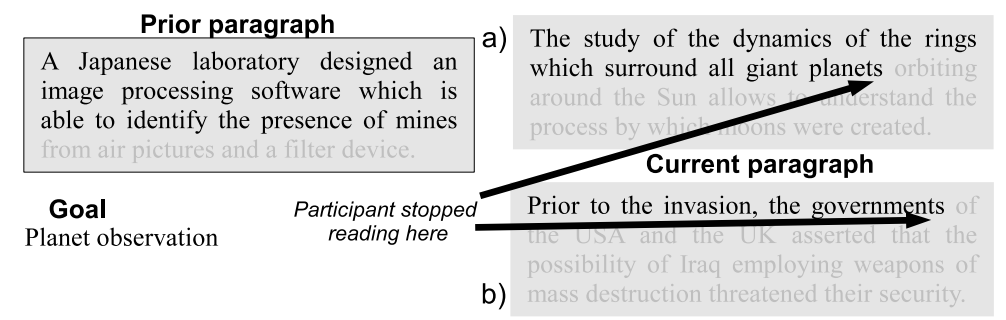

Fig. 1: Illustration of the 3 input data of the model: prior paragraph, goal, current paragraph. Prior paragraph has been processed partially. Current paragraph is abandoned before its end because enough information has been gathered and maybe due to a) a high-relatedness to the goal b) a low-relatedness to the goal.

This particular problem has been studied by Lee and Corlett (2003): participants were provided with a topic and a text, presented one word every second, and were asked to decide as quickly as possible if the text is about the given topic. However, we aim at studying a normal reading situation instead of presenting one word at a time. We will therefore rely on an eyetracker to identify the words processed. Figure 1 illustrates the situation we aim at modeling.

\section{Experiment}

In order to create and study a model, we designed an experiment to gather some data. This experiment was intended to emphasize the decision to stop reading a paragraph while two other pieces of information are stored in memory: another paragraph and the search goal. A set of 20 goals was created. Each one is expressed by a few words (e.g. mountain tourism). For each goal, 7 paragraphs were created (mean $=30.1$ words, $\sigma=2.9$ ), 2 of them being highly related to the goal, 2 of them being moderately related, and 3 of them being unrelated. We used Latent Semantic Analysis (LSA) (Landauer et al., 2007) to control the relatedness of a paragraph to the goal. Basically, LSA takes a large corpus as input and yields a high-dimensional vector representation for each word. It is based on a singular value decomposition of a word x paragraph occurrence matrix, where words occurring in similar contexts are represented by similar vectors. Such a vector formalism is very convenient to give a representation to sentences that were not in the corpus: the meaning of a new sentence is represented as a linear combination of its word vectors. Therefore, any sequence of words can be given a representation. The semantic similarity between two sequences of words (such as a goal and a paragraph) can be computed using the cosine function. The higher the cosine value, the more similar the two sequences of words. We trained LSA on a 24 million word general French corpus.

The experiment is composed of 20 trials, each one corresponding to a goal, in random order. In each trial, 2 paragraphs are presented together to the participant, as well as the goal (Fig. 2). The participant should select which paragraph 


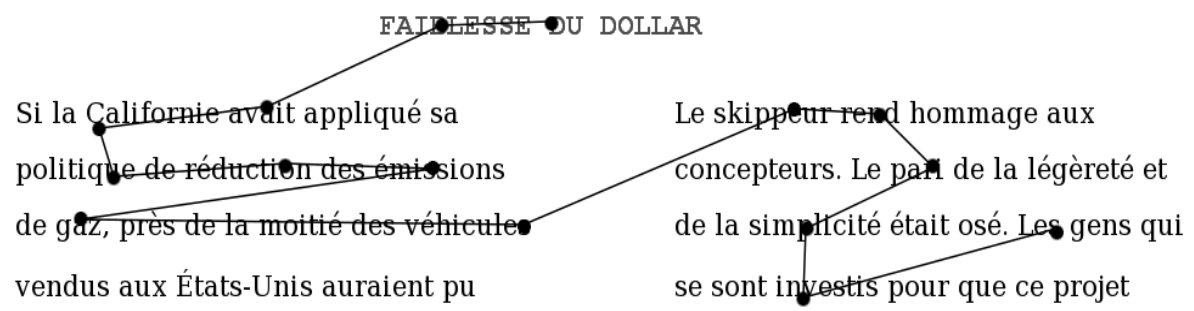

être concernés à moyen terme. existe peuvent être fiers.

Fig. 2: Example of material and scanpath.

is best related to the goal, by typing one key. The chosen paragraph is kept and the other is replaced by a new one. The participant should again select the most related to the goal. Then another paragraph replaces the one that was not selected and so on. This procedure is repeated until all 7 paragraphs of the current goal were displayed. Participants rated their confidence in their selection. Each participant was therefore exposed to $20 * 6=120$ pairs of paragraphs, and selected for each pair the paragraph which is most related to the goal. 22 participants participated in the experiment. Eye movements were recorded using a SR Research EyeLink II eye tracker. From these coordinates, saccades and fixations were determined, leading to an experimental scanpath, as shown in Fig. 2. The stimuli pages were generated with a software that stored the precise coordinates of each word on the screen. We wrote our experiment in Matlab, using the Psychophysics Toolbox (Brainard, 1997). Before trying to mimic eye movements, we had to predict which words were actually processed by participants in each fixation. It is known that the area from which information can be extracted during a single fixation extends from about 3-4 characters to the left of fixation to 14-15 characters to the right of fixation (Rayner, 1998). This area is asymmetric to the right and corresponds to the global perceptual span. Therefore, more than one word may be processed for a given fixation. In order to determine which ones were processed for each fixation, we used a window, sized according to Rayner (1998). He showed that the area from which a word can be identified extends to no more than 4 characters to the left and no more than 7-8 characters to the right of fixation and corresponds to the word identification span. Moreover, Pollatsek et al (1993) show that even if information of the next line is processed during a reading task, participants are not capable of getting some semantic information. Therefore, the size of our window is $4 \times 1$ characters to the left plus $8 \mathrm{x} 1$ characters to the right of the fixation point. Since the initial fixations in the beginning part of a word facilitate its recognition more than initial fixations toward the end of the word (Farid \& Grainger, 1996), we considered that a word is processed if at least the first third of it or the last two-thirds is inside the window. 


\section{Modeling}

The model should be able to predict the way a paragraph is processed, given a previous paragraph and a goal. For example, given the left paragraph of Fig. 2 and the goal, the model should be able to predict the way an average user would process the right paragraph (in this case the paragraph is processed partially).

Our method is therefore to consider the experimental scanpaths and for each participant's fixation to predict whether the paragraph would be abandoned or not. A very good model would predict an abandon at the same time the participant stopped reading. A bad model would abandon too early or too late.

Paragraphs can be examined several times by participants during a trial, but we restricted our analysis to first visits of the current paragraph. It is also worth noting that the previous paragraph is not necessary on the same stimuli page as the current paragraph. It could have been seen on the previous stimuli page. That is for instance the case of the left paragraph of Fig. 2 which has been processed with another paragraph in mind, seen on the previous stimuli page.

\subsection{Modeling Semantic Judgments}

Such a decision making model on paragraphs needs to be based on a model of semantic memory that would be able to mimic human judgments of semantic associations. We used LSA to dynamically compute the semantic similarities between the goal and each set of words that are supposed to have been fixated.

We assumed a linear exploration of words, although we know that this is not exactly the case in information search (Chanceaux et al., 2009).

\subsection{Effect of the Prior Paragraph}

The relatedness of the prior paragraph to the goal may play a role in the way the current paragraph is processed. We suspected that if the prior paragraph is not related to the goal, the current paragraph would be processed just to check whether it is relevant or not. The prior paragraph would not play a role in that case. However, if the prior paragraph is related to the goal, then the current paragraph may be processed with the idea of comparing it to the previous one.

We therefore analyzed two extreme cases: the words fixated in the prior paragraph are strongly related to the goal or they are not related at all to the goal. We used two thresholds of cosine similarity for that, which were set to 0.05 and 0.25 . Paragraphs whose semantic similarity with the goal falls in between were not considered. The first case is called $\mathrm{C} \mid \mathrm{S}$ (read the Current knowing that the previous one is Strong) and the second one is called $\mathrm{C} \mid \mathrm{W}$ (Current | Previous=Weak). We also analyzed cases when no prior paragraph exists, called $\mathrm{C} \mid 0$ (Current $\mid$ Nothing). Basic statistics show that in terms of number of fixations, fixation duration and the shape of the scanpath, $\mathrm{C}|\mathrm{W}=\mathrm{C}| 0$ and both are significantly different from $\mathrm{C} \mid \mathrm{S}$. It means that reading a paragraph while the other one is not related to the goal is similar to reading the very first paragraph, without information about a prior paragraph. 


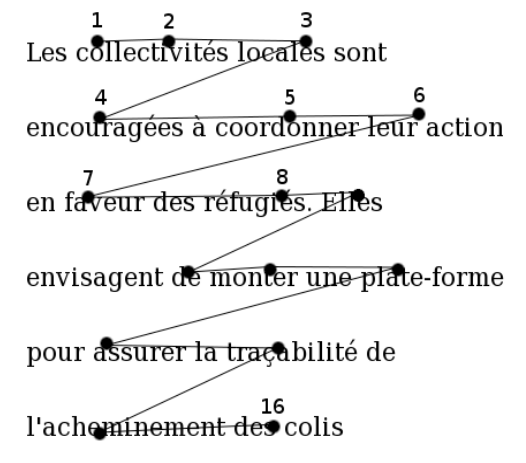

humanitaires.

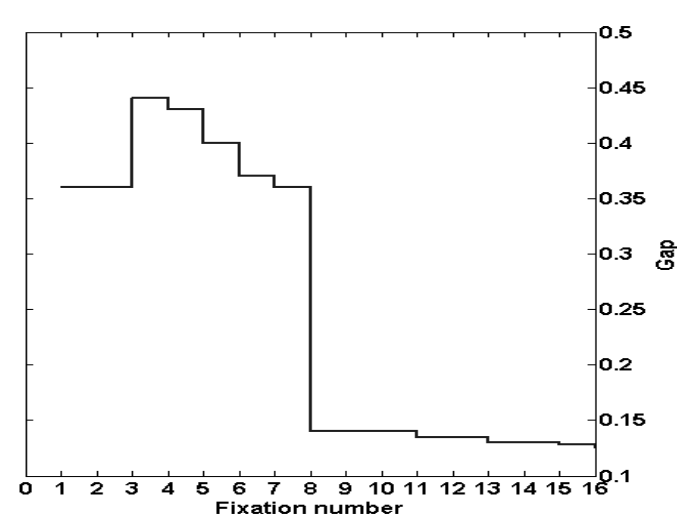

(b)

Fig. 3: a) Example of scanpath in the $\mathrm{C} \mid \mathrm{S}$ condition. b) Its Gap evolution.

Therefore we will only consider the case $\mathrm{C} \mid \mathrm{S}$ in this paper: reading a paragraph with another one in mind which is highly related to the goal.

\subsection{Modeling the Decision}

Two variables involved. We first looked for the variables which could play a role in the decision to stop reading a paragraph. Such a decision is made when the difference between the current $(c p)$ and the previous paragraph $(p p)$ is large enough to know for sure which one is the best. If they are too close to each other, no decision can be made and reading is pursued. The association to the goal $g$ is obviously involved in that perception of a difference between the two paragraphs. Therefore, we defined a variable called Gap $=\mid \operatorname{sim}($ words of $p p, g)-$ $\operatorname{sim}$ (words of $c p, g$ ) $\mid$ in which sim is the LSA cosine between the two vectors.

Gap changes constantly while a paragraph is processed since it depends on the words actually processed. When the two paragraphs are equally similar to the goal, that variable is zero. When one paragraph is much more associated to the goal than the other one, that variable has a high value. It can be easily calculated dynamically, after each word of the current paragraph has been processed. Consider for example Fig. 3a. Suppose that a prior paragraph has already been visited (paragraph and goal are not shown) and the sequence of words processed so far has led to a similarity sim $_{1}$ with the goal "associations humanitaires" of 0.62 . In the first two fixations on the current paragraph, only the word "collectivités" is supposed to have been processed according to our window-based prediction. Therefore in both cases Gap $=\mid$ sim $_{1}-$ $\operatorname{sim}($ "collectivités", "associations humanitaires") $\mid=0.62-0.26=0.36$.

During fixation 3, two extra words were processed leading to a new value of Gap $=\mid$ sim $_{1}-\operatorname{sim}($ "collectivités locales sont","associations humanitaires") $\mid=$ 0.44. In fixation 4, Gap $=\mid$ sim $_{1}-\operatorname{sim}$ ("collectivités locales sont encouragées à", "associations humanitaires") $\mid=0.43$. In fixation number 5, Gap $=\mid$ sim $_{1}-$ 


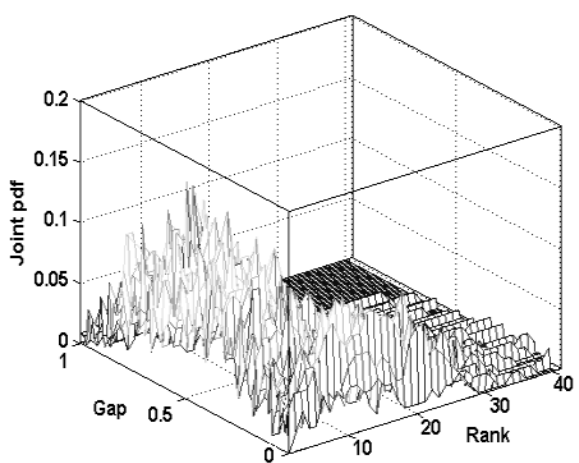

(a)

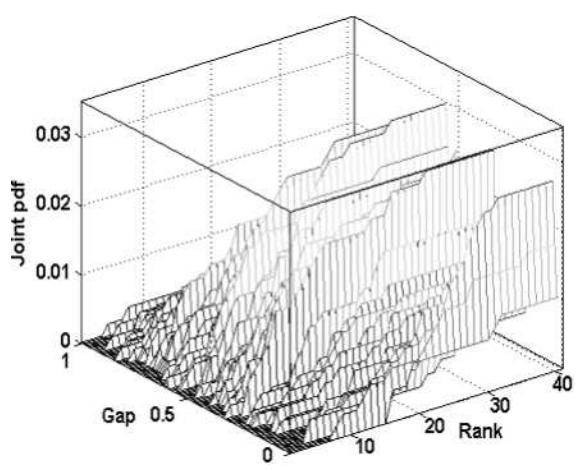

(b)

Fig. 4: a) Empirical "no-abandon" distribution $\hat{p}_{G R}(g, r \mid \overline{A b})$ and b) "abandon" distribution $\hat{p}_{G R}(g, r \mid A b)$ in the Gap $\times$ Rank space.

sim("collectivités locales sont encouragées à coordonner leur", "associations humanitaires") $\mid=0.40$. In fixation 8 , the Gap value dropped to 0.14 because of the word "réfugiés" which makes the LSA vector much more similar to the goal vector. Figure 3b shows the evolution of the Gap value along the fixations in the scanpath. This example illustrates that a high value of Gap may not directly induce the decision, in particular if it appears too early in the scanpath. We assume that the decision also depends on the number of words processed so far in the current paragraph. The more words processed, the higher the confidence in the perception of the difference between paragraphs. If only two or three words have been processed, it is less likely that Gap is accurate. Therefore, we assume that there should be a relationship between Gap and the number of words processed. The second variable is then $R a n k=$ number of words processed so far.

Abandon and no-abandon distributions. In order to study how the decision depends on these two variables, we computed two distributions in the Gap $\times$ Rank space of participant data: the distribution of the no-abandon cases and the distribution of the abandon cases. The goal is to learn the frontier between both cases in order to be able to predict if a sequence of words already processed is likely to lead to the abandon or the pursuance of the reading task. This work was done on two thirds of the data, in order to leave one third to test the model. Each participant fixation was associated to a point in the Gap $\times$ Rank space. Rank is a discrete measure between 1 and the maximum number of fixations in the data (93 in our case). Gap has been computed according to the previous formula, taking into account the words already processed in each paragraph as well as the goal and discretized into one of 100 bins, from 0 to 1.

The no-abandon distribution was computed by simply counting the number of fixations that did not lead to an abandon for each cell of the Gap $\times$ Rank grid. It concerns all fixations except the last one of each scanpath. 
The abandon distribution was built from all very last fixations of all scanpaths, including also subsequent ranks. For example, if a given participant on a given stimulus made 13 fixations, the first 12 were counted in the no-abandon distribution and the 13 th was counted in the abandon distribution. All virtual fixations from 14 to 93 , with the same gap value as the 13 th were also counted in the abandon distribution, because if the participant stopped reading at fixation 13 , he would have also stopped at fixation 14, 15, etc. The frontier between these two behaviors (continue or stop reading) is a curve in the Gap $\times$ Rank space. Depending on the location of any observation $(g, r)$ above or under the curve, the reader's behavior can be predicted. To find this frontier, a methodology based on a Bayesian classifier is used. Let us consider a classification problem with two classes: Abandon $(A b)$ and No-abandon $(\overline{A b})$. Given the posterior probabilities, which is the class of a two-dimensional observation $(g, r)$ in the Gap $\times$ Rank space? The decision rule is then:

$$
P(\overline{A b} \mid g, r) \underset{A b}{\stackrel{\overline{A b}}{\gtrless}} P(A b \mid g, r),
$$

with $P(\overline{A b} \mid g, r)=\frac{P(\overline{A b}) \times p_{G R}(g, r \mid \overline{A b})}{p_{G R}(g, r)}$, and $P(A b \mid g, r)=\frac{P(A b) \times p_{G R}(g, r \mid A b)}{p_{G R}(g, r)}$. Figures $4 \mathrm{a}$ and $4 \mathrm{~b}$ represent the two empirical class-conditional probability density functions respectively $\hat{p}_{G R}(g, r \mid \overline{A b})$ and $\hat{p}_{G R}(g, r \mid A b)$. We adopt a statistical parametric approach. By this way, data will be regularized since they are obviously affected by the noise inherent to acquisition and pre-processing.

In the next sections, the statistical model to estimate the density functions and the prior probabilities are explained in order to use the Bayesian classifier:

$$
P(\overline{A b}) \times p_{G R}(g, r \mid \overline{A b}) \underset{A b}{\stackrel{\overline{A b}}{\gtrless}} P(A b) \times p_{G R}(g, r \mid A b) .
$$

Parametric model for the "no-abandon" distribution. The class-conditional probability density function can be written as : $p_{G R}(g, r \mid \overline{A b})=p_{G \mid R}(g \mid R=$ $r, \overline{A b}) \times p_{R}(r \mid \overline{A b})$. Figure 5 (top, left) shows the empirical marginal distribution $\hat{p}_{R}(r \mid \overline{A b})$. As the Rank increases, the probability of not abandoning the paragraph decreases. This evolution was modeled with a sigmoid function $\varphi(r)=$ $\frac{P_{R M a x} \times\left(1+e^{-\alpha r_{0}}\right)}{1+e^{\alpha\left(r-r_{0}\right)}}$. There are actually only two parameters to fit because the integral is 1 .

Concerning the probability density function $p_{G \mid R}($.$) , the natural model (Fig.$ $4 a)$ is a Gaussian one whose parameters depend on the Rank value. The mean $\mu(r)$ and the standard deviation $\sigma(r)$ linearly decrease (Fig. 5, left column). The linear regressions are only performed up to the $\operatorname{Rank}=40$ since that $\hat{p}_{R}(r>$ $40 \mid \overline{A b})$ is close to zero and there is no more enough data. Then we have:

$$
p_{G \mid R}(g \mid R=r, \overline{A b})=\frac{A(r)}{\sqrt{2 \pi} \sigma(r)} e^{-\frac{(g-\mu(r))^{2}}{2 \sigma(r)^{2}}}, p_{R}(r, \overline{A b})=\varphi(r) .
$$

As the Gap value is between 0 and $1, A(r)$ is a normalization function to ensure that $p_{G \mid R}(g \mid R=r, \overline{A b})$ is a probability density function: $A(r)=F_{\mu, \sigma}(1)-$ 

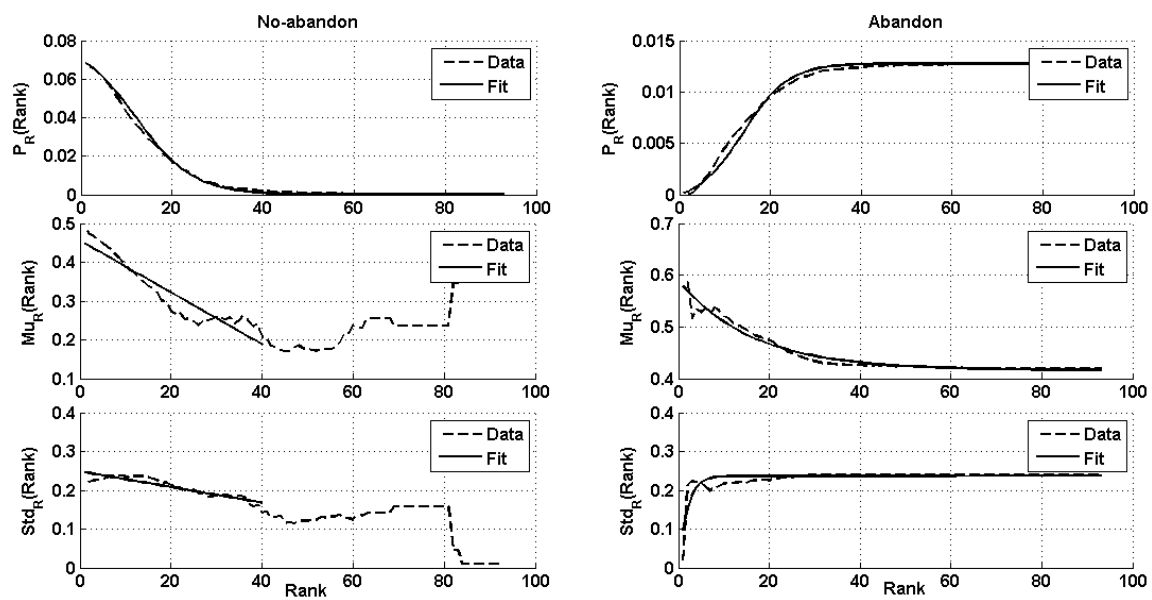

Fig. 5: Data and fitting of marginal distributions, mean and standard deviation for the "no-abandon" and "abandon" distributions.

$F_{\mu, \sigma}(0)$, with $F_{\mu, \sigma}($.$) being the repartition function of a Gaussian distribution$ with a mean $\mu$ and a standard deviation $\sigma$. We then obtained six independent parameters to model the complete "no-abandon" joint distribution (offset and slope for the sigmoid, and the two linear functions).

Parametric model for the "abandon" distribution. Following a similar approach the class-conditional pdf is written as : $p_{G R}(g, r \mid A b)=p_{G \mid R}(g \mid R=$ $r, A b) \times p_{R}(r \mid A b)$. The marginal pdf $\hat{p}_{R}(r \mid A b)$ was modeled with another sigmoid function $\varphi^{\prime}(r)$ (Fig. 5, top right). But here, it is an increasing function. At rank 0 , there is no abandon and at the maximal Rank value, all scanpaths have shown an abandon. The conditional distribution $\hat{p}_{G \mid R}(g \mid R=r, A b)$ is a Gaussian distribution with a mean $\mu^{\prime}(r)$ and a standard deviation $\sigma^{\prime}(r)$. The mean $\mu^{\prime}(r)$ exponentially decreases while the standard deviation $\sigma^{\prime}(r)$ exponentially increases (Fig. 5, right column). Equations of the pdf are the same as the previous case, but with a different set of functions $\left\{\varphi^{\prime}(r), \mu^{\prime}(r), \sigma^{\prime}(r)\right\}$ which gives us seven parameters $\left(2\right.$ for the $\varphi^{\prime}(r), 3$ for $\mu^{\prime}(r)$ and 2 for $\left.\sigma^{\prime}(r)\right)$ :

$$
p_{G \mid R}(g \mid R=r, A b)=\frac{A^{\prime}(r)}{\sqrt{2 \pi} \sigma^{\prime}(r)} e^{-\frac{\left(g-\mu^{\prime}(r)\right)^{2}}{2 \sigma^{\prime}(r)^{2}}}, p_{R}(r, A b)=\varphi^{\prime}(r) .
$$

Modeling the decision as the function of Rank and Gap. As these two class-conditional probabilities were modeled, for each (Rank, Gap) values, the problem is to decide if there is enough information to stop reading ("abandon" class), or to continue reading ("no abandon" class). This binary problem is solved thanks to the Bayesian classifier. To find this decision rule, we have now to estimate the prior probabilities such as : $P(A b)+P(\overline{A b})=1$. $P(A b)$ or $P(\overline{A b})$ is another parameter to learn from the data. The total number of learning param- 


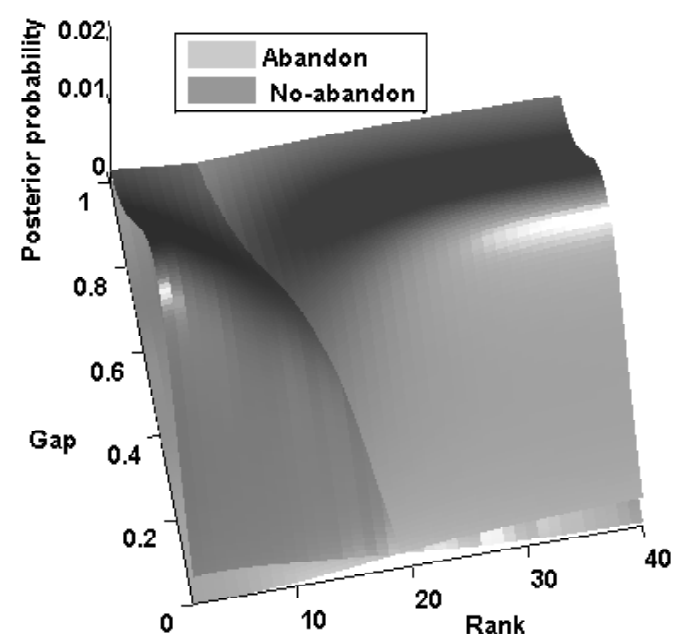

Fig. 6: The posterior probabilities $P(\overline{A b} \mid g, r)$ and $P(A b \mid g, r)$ in the $G a p \times$ Rank space.

eters is then $14(6+7+1)$. The decision rule is then:

$P(\overline{A b}) \times p_{G \mid R}(g \mid R=r, \overline{A b}) \times p_{R}(r \mid \overline{A b}) \underset{A b}{\stackrel{\overline{A b}}{\gtrless}} P(A b) \times p_{G \mid R}(g \mid R=r, A b) \times p_{R}(r \mid A b)$.

\section{Model Learning}

Figure 6 shows the two posterior probabilities $P(\overline{A b} \mid g, r)$ and $P(A b \mid g, r)$ after learning in order to represent the decision frontier between the two classes. The two prior probabilities are $P(A b)=0.84$ and $P(\overline{A b})=0.16$. As Fig. 6 shows, the intersection is oblique which is what was expected, from a cognitive point of view. Rank and Gap are dependent on each other: at the beginning of processing the paragraph (low values of the Rank), there should be a high difference between the two paragraphs to make the decision. However, after more fixations have been made, that difference could be lower to decide to abandon the paragraph.

For instance, at rank 10, a Gap of .86 is necessary to stop reading, whereas at rank 15, a value of .42 is enough. The frontier is rather linear and can be approximated by the following equation in the Gap $\times$ Rank space:

$$
G a p_{0}=-0.090 \times \text { Rank }+1.768 .
$$

That equation was included in the computational model. That model constantly computes the Gap value while it is moving forward in the text, increasing the Rank value. As soon as the current Gap value is greater than $G a p_{0}$, the decision is to stop reading the paragraph.

In order to test the model, we ran it on the remaining one third of the data. For each fixation in this testing set, the model decides either to leave or not 
to leave the paragraph. If the model did not leave at the time the participant stopped reading, simulation is pursued with the next rank and with the same value of the gap, and so on until the decision is made. The average difference between the ranks at which model and participant stopped reading was computed. We got a value of 6.58 ( $\mathrm{SE}=0.29)$. To assess the significance of that value, we built a random model which stops reading after each fixation with probability $p$. The smallest average difference between participants' and model's ranks of abandoning was $11.47(\mathrm{SE}=0.45)$ and was obtained for $p=0.20$. Our model therefore appears to be much better than the best random model.

\section{Conclusions}

We presented a model which predicts the sequence of words that are likely to be fixated before a paragraph is abandoned given a search goal. Two variables seem to play a role: the rank of the fixation and the difference of semantic similarities between each paragraph and the search goal. We proposed a simple linear threshold to account for that decision. Our model will be improved in future work. In particular, we aim at considering a non linear way of scanning the paragraph, using another model of eye movements (Lemaire et al., 2011). We also plan to tackle more realistic stimuli as well as extending that approach to consider other decisions involved in Web search tasks.

\section{References}

Brainard, D. H.: The Psychophysics Toolbox, Spatial Vision 10(1997) 433-436.

Brumby, D. P., Howes, A.: Good enough but I'll just check: Web-paged search as attentional refocusing. In Proc of the 6th ICCM Conference (2004) 46-51.

Chanceaux, M., Guérin-Dugué, A., Lemaire, B., Baccino, T.: A model to simulate Web users' eye movements. In Proc of the 12th INTERACT Conference, LNCS 5726, Berlin: Springer Verlag, (2009) 288-300.

Farid, M., Grainger, J. How initial fixation position influences word recognition: A comparison of French and Arabic. Brain \& Language, 53, (1996) 351-368.

Landauer, T., McNamara, D., Dennis, S., Kintsch, W.: Handbook of Latent Semantic Analysis. Lawrence Erlbaum Associates, (2007).

Lee, M.D., Corlett, E.Y. Sequential sampling models of human text classification. Cognitive Science, 27(2), (2003) 159-193.

Lemaire, B., Guérin-Dugué, A., Baccino, T., Chanceaux, M., Pasqualotti, L.: A cognitive computational model of eye movements investigating visual strategies on textual material. In L. Carlson, C. Hölscher and T. Shipley (Eds.) Proc of the Annual Meeting of the Cognitive Science Society, (2011) 1146-1151.

Pirolli, P., \& Fu, W. SNIF-ACT: a model of information foraging on the world wide web. In P. Brusilovsky, A. Corbett, \& F. de Rosis (Eds.), 9th ICUM (2003) 45-54.

Pollatsek, A., Raney, G. E., LaGasse, L., \& Rayner, K.: The use of information below fixation in reading and in visual search. Can J Psychol 47, (1993) 179-200.

Rayner, K. Eye movements in reading and information processing: 20 years of research. Psychological Bulletin 124(3), (1998) 372-422. 